\title{
Fotemustine as second-line treatment for recurrent or progressive glioblastoma after concomitant and/or adjuvant temozolomide: a phase II trial of Gruppo Italiano Cooperativo di Neuro-Oncologia (GICNO)
}

\author{
Alba A. Brandes · A. Tosoni · E. Franceschi • V. Blatt \\ A. Santoro · M. Faedi · P. Amistà · M. Gardiman • \\ R. Labianca $\cdot$ C. Bianchini $\cdot$ M. Ermani $\cdot$ M. Reni \\ Received: 24 October 2008 / Accepted: 5 January 2009 / Published online: 24 January 2009 \\ (C) Springer-Verlag 2009
}

\begin{abstract}
Background Standardized salvage treatment has not yet proved effective in glioblastoma multiforme (GBM) patients who receive prior standard radiotherapy plus concomitant and adjuvant temozolomide.

Methods Patients with progressive GBM after radiotherapy plus concomitant and/or adjuvant temozolomide received three-weekly doses $\left(100-75 \mathrm{mg} \mathrm{m}^{2}\right)$ of fotemustine followed, after a 5-week rest, by fotemustine $\left(100 \mathrm{mg} \mathrm{m}^{2}\right.$ ) every 3 weeks for $\leq 1$ year.

Results Forty-three patients (29 M, 14 F; median age 51 years, range 34-68; median KPS 90) were enrolled. Progression-free survival at 6 months (PFS-6) was $20.9 \%$ (95\% CI: 9-33\%); three patients (7.1\%) had partial response (PR); 15 (34.9\%), disease stabilization (SD). The median survival was 6 months $(95 \% \mathrm{CI}: 5-7)$. MGMT
\end{abstract}

A. A. Brandes $(\bowtie) \cdot$ A. Tosoni · E. Franceschi

Department of Medical Oncology,

Ospedale Bellaria-Maggiore, Via Altura 3,

40139 Bologna, Italy

e-mail: alba.brandes@yahoo.it

V. Blatt

Research and Development Unit,

Azienda Ospedale-Università di Padova, Padova, Italy

\section{A. Santoro}

Department of Medical Oncology,

Istituto Clinico Humanitas, Rozzano, Italy

M. Faedi

Department of Medical Oncology,

Ospedale Bufalini, Cesena, Italy

P. Amistà

Department of Neuroradiology,

Ospedale Santa Maria della Misericordia, Rovigo, Italy promoter status was methylated in $8(18.6 \%)$ and unmethylated in $26(60.5 \%)$ and not assessable in $9(20.9 \%)$ patients, respectively. Disease control was $75 \%$ versus $34.6 \%$ in methylated and unmethylated $M G M T$ patients $(P=0.044)$; no significant difference was found between groups for PFS-6 and survival. Grade 3 and 4 thrombocytopenia and neutropenia were observed in 20.9 and $16.3 \%$ of patients, during the induction phase, and in 0 and $9.5 \%$ patients during the maintenance phase, respectively.

Conclusions The findings of the present trial, that evaluate fotemustine in a homogeneous population, may represent a new benchmark for nitrosourea activity. Moreover, this is the first study to evaluate correlation between MGMT promoter status and outcome of fotemustine for relapsing GBM previously treated with radiotherapy and temozolomide.

\author{
M. Gardiman \\ Department of Pathology, \\ Azienda Ospedale-Università di Padova, Padova, Italy \\ R. Labianca \\ Department of Medical Oncology, \\ Ospedali Riuniti, Bergamo, Italy \\ C. Bianchini \\ Scientific Department, Italfarmaco, Milan, Italy \\ M. Ermani \\ Department of Neurosciences, Statistics and IT Unit, \\ Azienda Ospedale-Università di Padova, Padova, Italy \\ M. Reni \\ Department of Oncology, \\ San Raffaele Scientific Institute, Milan, Italy
}


Keywords Glioblastoma multiforme $\cdot$ Second-line chemotherapy $\cdot$ Recurrence $\cdot$ Fotemustine $\cdot M G M T$. Clinical trial

\section{Introduction}

Worldwide, glioblastoma multiforme (GBM) is the most frequent primary brain tumor in adults, accounting for 15$20 \%$ of intracranial tumors and $50 \%$ of gliomas [6]. The standard treatment for newly diagnosed GBM with temozolomide (TMZ), concomitant, and adjuvant to radiotherapy provides a significant increase in overall survival with respect to radiotherapy alone [27]. However, nearly all patients treated for GBM faced recurrence. None of the several drug regimens reported in literature substantially delays disease progression [2]. However, promising results have recently been obtained using novel agents, and new schedules or synergic combinations [23, 29, 30]. However, the findings reported following these new approaches require further confirmation based on results obtained in patients who have a longer follow-up.

Nitrosoureas, used alone or in combination with other agents, are still used as standard second-line chemotherapy, and are considered the standard arm in randomized phase II studies for experimental therapy. Moreover, it has not been demonstrated that nitrosourea-based polychemotherapy is anymore effective against GBM than single agent chemotherapy. Fotemustine (FTMS), a third generation chloroethylnitrosourea containing a phosphoalanine carrier group, is grafted to the nitrosourea radical. Thanks to the phosphoalanine group contained, the drug is highly lipophilic; its octanol/water partition coefficient being within a range that is more satisfactory than the ranges obtained with other nitrosoureas, such as carmustine (BCNU) and lomustine (CCNU). FTMS is able to cross the blood-brain barrier [16, 18]; in vitro and in vivo studies have shown that FTMS has a marked anti-neoplastic activity on human GBM and medulloblastoma cell lines [9, 10]. In their phase I study on 22 GBM patients, Khayat et al. [14] specified the dose of FTMS to be used in clinical practice: $100 \mathrm{mg} \mathrm{m}^{2}$ for 1 -h infusions, conducted on days 1,8 , and 15 (induction), to be repeated after 4-5 weeks (hematological recovery) every 21 days (maintenance). However, in the literature, there is little information on activity and toxicity of this regimen.

The aim of the present phase II study on patients with recurrent or progressive GBM, who were uniformly treated with prior radiotherapy and TMZ, was to evaluate the effect of FTMS on progression-free survival at 6 months (PFS-6), response, toxicity and any correlation with O6-methylguanine-DNA methyltransferase (MGMT) gene promoter methylation status.

\section{Patients and methods}

Eligibility

The criteria for eligibility were GBM recurrent or progressive after surgery and TMZ concomitant with and/or adjuvant to radiotherapy, proven by MRI or CT scans at least 3 months following the radiotherapy end or by two consecutive tests. Measurable disease with contrast enhancement using MRI or CT scans, tested within 2 weeks before study treatment start. In case of re-surgery before chemotherapy start, residual measurable disease with contrast enhancement must be proven by MRI or CT scans, performed within 3 days after surgery. At least one unidimensionally measurable lesion of $\geq 2 \mathrm{~cm}$ in diameter by MRI. Stable or decreasing dose of corticosteroids for at least 2 weeks before patient's enrollment. At least 4 weeks following chemotherapy with TMZ. Other inclusion criteria were age $\geq 18$ and $\leq 70$ years; Karnofsky performance status $\geq 60$; adequate bone marrow reserve (absolute neutrophils count $>1.5 \times 10^{9} \mathrm{~L}^{-1}$; platelets $>100 \times 10^{9} \mathrm{~L}^{-1}$; hemoglobin $>10 \mathrm{~g} \mathrm{dL}^{-1}$ ); normal renal and liver function (serum creatinine $<1.25 \times$, upper limit of the normal range (ULN); BUN $<25 \mathrm{mg} \mathrm{dL}^{-1}$; serum bilirubin $\leq 1.25 \times \mathrm{ULN}$; AST and $\mathrm{ALT} \leq 1.5 \times \quad \mathrm{ULN} ;$ alkaline phosphatase $\leq 2 \times \quad$ ULN; remaining life expectancy $\geq 3$ months. Patients with active infections or other uncontrolled diseases, psychiatric disturbances and/or a previous history of cancer (except for resected non-melanoma skin cancer or carcinoma-in situ of the uterine cervix), were considered ineligible.

All the histological specimens obtained at first diagnosis were reviewed by the coordinating center of Azienda Ospedaliera of Padova (M.G. Department of Pathology) and the diagnosis of GBM was confirmed according to the criteria specified in 2007 WHO central nervous tumor classification.

The study, approved by the Institutional Ethics Committees of all participating centers, was conducted according to the principles of the declaration of Helsinki and the rules of good clinical practice.

All patients signed a form giving their fully informed consent to participate in the study.

\section{Treatment schedule}

In line with phase I study protocol and with licensing instructions of the drug, FTMS $100 \mathrm{mg} \mathrm{m}^{2}$ was administered i.v. over $1 \mathrm{~h}$ weekly for three consecutive weeks (induction therapy), followed after 5 weeks by one infusion of FTMS $100 \mathrm{mg} \mathrm{m}^{2}$ every 3 weeks (maintenance therapy) for up to 1 year, unless disease progression or unacceptable toxicity was observed. If, due to toxicity, treatment suspension was prolonged by more than 2 weeks beyond the next scheduled cycle of treatment planned, the patient was 
permanently withdrawn from the study. Based on the most severe toxicity experienced since the last cycle, the subsequent dose was reduced to $75 \%$ in the presence of grade 3 or 4 platelet toxicity, grade 4 neutrophils or white blood cells or hemoglobin toxicity. In cases of non-hematologic toxicity, chemotherapy was delayed until recovery to grade 1 , for a maximum of 2 weeks (after which the patient was withdrawn from the study). In cases of recovery to grade 1 after grade 3 or 4 toxicity, a dose of $75 \%$ the treatment dose was administered.

After the inclusion of the first three patients who experienced grade 4 thrombocytopenia following induction therapy, the protocol was amended to reduce FTMS dosage during induction therapy to $75 \mathrm{mg} \mathrm{m}^{2}$. In fact, this life-threatening toxicity was considered unacceptable in the palliative setting of salvage therapy of GBM patients.

\section{Efficacy measures and toxicity monitoring}

Progression-free survival (PFS) was measured as from the initiation of FTMS to progression or death due to any cause or last follow-up assessment, whichever comes first. Overall survival (OS) was measured as from the start of FTMS to death for any reason, or last follow-up assessment. In this intent to treat study, data on all registered patients who met the main inclusion criteria were included in the statistical analysis.

Evaluation of response, conducted in all patients, included clinical and neurological examinations and MRI or CT neuro-imaging according to Macdonald's criteria [17]. The first evaluation was made after the induction phase (7 weeks after the first study drug administration); thereafter evaluations were made every two cycles during the study treatment ( 6 weeks) and every 3 months during the follow-up period, or earlier if indicated. Neurological status was assessed by considering signs and symptoms possibly correlated with progression, as compared to the previous examination; each variation in daily corticosteroids dosage was recorded.

Responses were confirmed as complete (CR), partial (PR) and stable (SD) if they were constant at subsequent scans obtained at least 4 weeks apart from each other. An independent central review of CT and MRI scans was made for all patients.

All adverse events were recorded and graded according to the common toxicity criteria of the National Cancer Institute, version 3.0. (http://ctep.cancer.gov/forms/CTCAEv3.pdf).

DNA extraction and methylation-specific polymerase chain reaction

MGMT promoter methylation analysis was performed on tissue taken from the primary surgery specimen before radiotherapy and TMZ.
DNA from $10 \mu \mathrm{m}$ paraffin sections of cerebral lesion was modified by sodium bisulfite, which converts unmethylated cytosine to uracil, according to the procedure of Herman et al. [13]. Modified DNA was submitted for methylation-specific PCR (MSP) following a nested-PCR protocol [21]. Since the quality of DNA obtained from formalin-fixed paraffin-embedded tumor tissue affects the success rate of MSP, in some cases MGMT methylation status was determined by a different nested-MSP approach, with a first pair of primers to obtain smaller amplicons (129 bp), for which forward and reverse primers have been described $[21,28]$.

End points and statistical analysis

Data from all patients, who received at least one drug delivery and for whom at least one tumoral evaluation was performed, were included in the response analysis.

The primary efficacy endpoint of the study was the percentage of patients free from disease progression at 6 months (PFS-6). Drug activity was evaluated following a one-stage Fleming study design for determination of response rates based on a single-treatment group. A sample size of 40 patients was estimated using exact binomial method and assuming: one-tailed $\alpha$ equal to $0.1,(1-\beta)$ equal to 0.9 and $\pi<0.1$ (null hypothesis) versus $\pi \geq 0.25$ (alternative hypothesis), where $\pi$ was the observed 6-month disease progression-free probability. If seven or more patients were evaluated as PFS-6, it was assumed that the drug was active.

Secondary objectives were the rate of best observed response, defined as the best response during the treatment and evaluated with Macdonald's criteria [17]; duration of objective response and stabilization; duration of complete response; time to disease progression, overall survival, toxicity, and evaluation of $M G M T$ methylation and the correlation with clinical outcome. All patients receiving the study drug were included in the safety analysis. Median time to progression (mTTP) and median survival were also estimated with associated 95\% CI. PFS-6 and OS were calculated using the Kaplan-Meier method; differences in PFS-6 and OS were compared using the log rank test for statistical significance.

\section{Results}

Patients' characteristics

From April 2005 to May 2006, 43 patients (29 males; median age: 51 years, range: $34-68$ years; median: KPS 90) were enrolled in the study. The demographic and clinical characteristics of patients are outlined in Table 1. Each 
Table 1 Patients' characteristics

\begin{tabular}{lcc}
\hline & Number of patients & $\%$ \\
\hline Sex & 29 & 67 \\
Male & 14 & 33 \\
Female & & \\
Age & $51(34-68)$ & \\
Median years (range) & $90(70-100)$ & \\
Karnofsky performance status (KPS) & \\
Median (range) & & 65 \\
Extent of resection & 28 & 28 \\
Macroscopically radical & 12 & 7 \\
Partial & 3 & 100 \\
Biopsy & 43 & 18.6 \\
Radiotherapy/TMZ & & 60.5 \\
MGMT status & 8 & 20.9 \\
Methylated & 26 & 69.8 \\
Unmethylated & 9 & 30.2 \\
Unknown & 13 & \\
FTMS initiation and time to TMZ treatment completion \\
Within 3 months & 30 & \\
Beyond 3 months & & \\
\hline
\end{tabular}

patient had completed external-beam radiation therapy (60 Gy/30 F) concurrent and/or followed by adjuvant TMZ. Median number of TMZ cycles was 5 (range 1-23). No patient underwent a second surgical procedure at the time of progression following TMZ administration. Median time between TMZ treatment completion and FTMS start was 1.5 months (range 1-43). Thirty patients (69.8\%) started FTMS within 3 months from TMZ last administration. At time of FTMS initiation 26 patients $(60.5 \%)$ were on enzyme-inducing antiepileptic drugs (EIAEDs), while 11 patients $(25.6 \%)$ were on non-EIAEDs and 6 patients (13.9\%) were not on antiepileptic drugs. The median duration of follow-up was 7.4 months (range 1.2-22.7). In 39 of the 43 patients enrolled in the trial, enough histological material was obtained for evaluation of MSP. MSP was assessable on 34 of these 39 patients. MGMT promoter status was methylated in $8(18.6 \%)$ and unmethylated in $26(60.5 \%)$ and not assessable in $9(20.9 \%)$ patients, respectively.

Progression-free survival

Nine patients (20.9\%; 95\% CI: 9-33\%) were PFS-6 and the median PFS was 1.7 months.

The percentages of PFS-6 with MGMT promoter methylated or unmethylated status were $25 \%$ (95\% CI: $7.5-83 \%$ ) and $19.2 \%$ (95\% CI: 8.7-42.3\%), respectively. PFS-6 in the population $(n=40)$ treated after the amendment was
$22.5 \%$, not significantly different with the entire population of 43 pts.

No significant differences were found between median PFS, evaluated using the log rank test, in relation to age $(P=0.89)$, KPS $(P=0.33)$, and $M G M T$ promoter methylated or unmethylated status $(P=0.15)$. No significant influence of type of antiepileptic drug was seen, being PFS-6 $26.9 \%$ and $11.7 \%$ in patients on EIAEDS and on nonEIAEDS plus not on antiepileptic drugs, respectively $(P=0.28)$.

Patients that initiated FMTS at least 3 months after TMZ completion showed a significantly higher PFS-6, (30.7 vs. $16.7 \%$ ) than patients who initiated FTMS immediately after TMZ completion $(P=0.034)$.

\section{Response}

Among the 43 assessable patients, 3 had partial responses (7.1\%, 95\% CI: $0-15 \%)$, and 15 stable disease $(34.9 \%$, 95\% CI: $21-49 \%$ ). Disease control rate (SD+PR) in the population treated after the amendment was $42.5 \%$, not significantly different with the entire population. All responses were confirmed by an independent centralized review, and stable or decreased steroid dosage was confirmed in all patients at the time of recording response. Median duration of response was 9.1 months (95\% CI: 1.7-16.4), and median duration of disease stabilization was 5 months (95\% CI: 1.2-8.9). Disease control rate was significantly greater in methylated and unmethylated $M G M T$ patients, 75 and $34.6 \%(P=0.044)$, respectively; and in patients who started FTMS at least 3 months after TMZ administration had been concluded (76.9 vs. $26.7 \%, P=0.002$ ).

No significant influence of type of antiepileptic drug was seen, being disease control rate 42.3 and $41.2 \%$ in patients on EIAEDS and on non-EIAEDS plus non on antiepileptic drugs, respectively $(P=0.98)$.

\section{Overall survival}

The median overall survival was 6 months (95\% CI: 5-7). The median overall survival of the population treated after the amendment was 6 months. No statistical difference has been found between patients with methylated and those with unmethylated MGMT promoter status: being 6 months (95\% CI: 0-14.2) versus 5.5 months (95\% CI: 4.2-6.8).

The median overall survival for patients who started FTMS at least 3 months after TMZ administration had been concluded was 8.4 months (95\% CI: 2.6-14) versus 5.4 months (95\% CI: 4.2-6.5) for patients who initiated FTMS immediately after TMZ completion $(P=0.022)$.

The percentage of patients alive at 6 months was $51 \%$ (95\% CI: 38-68\%), without difference between patients with methylated or unmethylated $M G M T$ promoter status 
62.5\% (95\% CI: $36.5-100 \%$ ) versus $46 \%$ (95\% CI: $30.5-$ $70 \%)$. Only disease control rate obtained with FTMS was significantly correlated with survival $(P=0.002)$.

Treatment and toxicity

All 43 patients completed the induction phase as planned. After induction phase grade 3-4 thrombocytopenia and neutropenia were documented in $9(20.9 \%)$ and 7 out 43 patients $(16.3 \%)$, respectively; grade 3-4 lymphopenia was found in four patients $(9.3 \%)$. The study was emended after the first three patients by decreasing the FTMS induction dose from $100 \mathrm{mg} \mathrm{m}^{2}$ once a week for 3 weeks to $75 \mathrm{mg} \mathrm{m}^{2}$. Of note, the first three patients that received induction therapy at the dosage of $100 \mathrm{mg} \mathrm{m}^{2}$ aged 48, 51, and 62 years, had a KPS of 100, 100, and 80, started FTMS 30, 40, and 95 days after TMZ completion, respectively, and did not have significant comorbidities to justify increased toxicities.

The grade 3-4 thrombocytopenia reported for the induction phase in the 40 patients treated after this amendment was $15 \%$.

Twenty-one (49\%) patients started maintenance chemotherapy and received a median of two cycles (range 1-14). The main reason for not beginning maintenance therapy after the induction part was disease progression. Only one patient, with prolonged grade 2 neutropenia, discontinued therapy due to toxicity after the induction phase. The toxicity of the maintenance phase was grade 3 leukopenia and grade 4 neutropenia in 14 and $9.5 \%$ of the patients, respectively; no patient experienced grade 3-4 thrombocytopenia (Table 2). The most commonly reported grade 3-4 nonhematological toxicity were nausea and vomiting in two $(4.6 \%)$ patients and transaminase elevation in four patients $(9.3 \%)$. Pneumonia was reported in one patient $(2.3 \%)$.

\section{Discussion}

TMZ concomitant and adjuvant to radiotherapy, which has become the standard treatment for newly diagnosed GBM patients, prolongs overall and progression-free survival more effectively than radiotherapy alone [27]. Consequently, first-line chemotherapy has become more homogeneous than it was in the past. However, while the outcome of patients with newly diagnosed GBM has improved worldwide, recurrence continues to be virtually inevitable. The choice of second-line chemotherapy is therefore of utmost importance in the large number of patients who continue to have a satisfactory PS and are willing to receive further treatment, nitrosourea being the most widely used therapeutic option. However, the real impact of this salvage chemotherapy in terms of activity, disease control, and
Table 2 Hematological toxicities during and after induction and during the maintenance phase

\begin{tabular}{llll}
\hline $\begin{array}{l}\text { Adverse } \\
\text { Event }\end{array}$ & $\begin{array}{l}\text { Induction } \\
(n=43) \\
{[\%(\text { Pts/Total })]}\end{array}$ & $\begin{array}{l}\text { Induction after } \\
\text { amendment } \\
(n=40) \\
{[\%(\text { Pts/Total })]}\end{array}$ & $\begin{array}{l}\text { Maintenance } \\
(n=21) \\
{[\%(\text { Pts/Total })]}\end{array}$ \\
\hline $\begin{array}{l}\text { Thrombocytopenia } \\
\text { Grade 3 }\end{array}$ & $11.6(5 / 43)$ & $12.5(5 / 40)$ & $0.0(0 / 21)$ \\
Grade 4 & $9.3(4 / 43)$ & $2.5(1 / 40)$ & $0.0(0 / 21)$ \\
Leukopenia & & & $14.3(3 / 21)$ \\
Grade 3 & $7.0(3 / 43)$ & $5.0(2 / 40)$ & $0.0(0 / 21)$ \\
Grade 4 & $2.3(1 / 43)$ & $2.5(1 / 40)$ & $0.0(0 / 21)$ \\
Neutropenia & & $10.0(4 / 40)$ & $9.5(2 / 21)$ \\
Grade 3 & $9.3(4 / 43)$ & $5.0(2 / 40)$ & $14.3(3 / 21)$ \\
Grade 4 & $7.0(3 / 43)$ & & $0.0(0 / 21)$ \\
Lymphopenia & $7.0(3 / 43)$ & $7.5(3 / 40)$ & \\
Grade 3 & $2.5(1 / 40)$ & \\
Grade 4 & $2.3(1 / 43)$ & &
\end{tabular}

toxicity after TMZ failure is still largely unknown. The present study is the first trial to evaluate correlation between MGMT methylation status (assessable in 79\% of patients) and outcome of nitrosourea-based chemotherapy for progressing/relapsing glioblastoma previously treated with radiotherapy and TMZ in the adjuvant setting. Data on the outcome of fotemustine administration, used as secondline treatment in GBM patients were reported by Scoccianti et al. [26], and Fabrini et al.[8]; the authors reported a similar PFS-6 rates (48 and 52\%, respectively), disease control rate (48 and $62 \%$, respectively), grade 3-4 hematological toxicities (14.8 and $10 \%$, respectively).

The results from these series seem to be better than ours both in terms of outcome and of toxicity. However, comparison across trials is always challenging. Moreover, the lack of MGMT methylation status data in the other FTMS studies and the small number of patients included in these three studies might justify the differences, and highlight the relevance of performing larger prospective trials in this patient population.

We decided to modify the fotemustine dose recommended in a prior phase I trial [14] and in other studies on glioma or melanoma patients [1, 15, 19, 20, 25], as grade 4 thrombocytopenia was observed after induction therapy in the first three patients and this life-threatening toxicity was considered unacceptable in the palliative setting of salvage therapy of GBM patients. This is consistent with the previous observation that hematological toxicity is increased by prior chemotherapy [24]. Moreover, the following 40 patients, treated with the reduced dosage of FTMS during induction phase, experienced a $15 \%$ of grade 3-4 thrombocytopenia and neutropenia. 
Maintenance fotemustine showed hematological toxicities similar to those showed by BCNU delivered at firstrelapse (grade 3-4 neutropenia and thrombocytopenia in 10 and $8 \%$ of patients, respectively) [3]. However, no cumulative dose limit for fotemustine was found by us, whereas the use of BCNU is complicated by pulmonary toxicity (G4 toxicities occurring in $5 \%$ of cases [3]), which severely compromises quality of life, and life expectancy and leads to discontinuation of therapy (10\%).

Fotemustine administration was found to be more feasible and tolerable than PCV treatment, incurring fewer cases of discontinuation due to toxicity ( 2.3 vs. $43 \%$ ) [5]. In terms of disease control, fotemustine yielded a PFS- 6 of $21 \%$, which met the primary efficacy end point of the study by confirming the drug activity.

Apparently promising results (response rate, 57\%; PFS6, 46\%; 6-month overall survival, $77 \%$ ) were recently reported following the use of combined irinotecan and bevacizumab [29]. However, these drugs have not yet been registered by FDA or EMEA and nor are they available worldwide, nitrosoureas continuing to be the most commonly used second-line standard therapy.

Other interesting approaches include TMZ re-challenge, which was recently investigated by Perry et al. In their retrospective analysis, the authors showed that TMZ re-challenge with a continuous $50 \mathrm{mg} \mathrm{m}^{-2}$ daily schedule is an intriguing approach, especially for patients with recurrence after completion of TMZ administration concurrent with and adjuvant to radiotherapy: GBM patients failing during the first 3-6 months of adjuvant therapy (B1); GBM patients failing after more than 6 months of therapy (B2); GBM patients who recurred after stopping treatment (B3). PFS-6 rates were $28.6 \%$ (B1), 9.5\% (B2), 30.4\% (B3) [22]. Our findings are in line with these observations: patients in our series who initiated FMTS at least 3 months after completion of TMZ administration had a significantly higher PFS-6, (30.7 vs. $16.7 \%$ ) than patients who initiated FTMS while still on TMZ, $P=0.034$ ).

$M G M T$ promoter methylation was found to be an independent favorable prognostic factor, irrespective of treatment, in newly diagnosed GBM patients [12]. Conversely, little information is available on the trend of MGMT expression during tumoral progression, and after different chemotherapeutic treatments. In the present study, an analysis was made of the correlation between clinical outcome after fotemustine and MGMT promoter methylation status; to our knowledge, ours is the first study to analyze the correlation between $M G M T$ promoter methylation status and second-line treatment. Adequate paraffin embedded tumor tissue was available in a higher percentage of patients than in other MSP studies (79 vs. 59-67\%) [11, 12]. The percentage of methylated patients found in our study was clearly smaller than those reported in other series given upfront and/or salvage therapy (24 vs. 40-47\%) [7, 12]. Moreover, we found that disease control rate was 75 and $34.6 \%$ in methylated and unmethylated patients $(P=0.044)$. On the other hand, no significant difference was found between groups for PFS-6 and survival; this finding, which is in line with that reported in other series of heterogeneously pre-treated patients who underwent salvage therapy [4], may reflect or a different pattern of resistance at progression, or a change in MGMT status at progression. A trend toward prolonged PFS-6 was observed, the lack statistical significance probably reflecting limited statistical power due to the relatively small number of cases in the present study, which precluded the demonstration of this secondary endpoint. It is important to bear in mind that $M G M T$ methylation analysis was not made in trials that reported more favorable results [29]. For a reliable assessment of the role of this variable at the time of salvage, a prospective report should be undertaken to evaluate $M G M T$ methylation status and, hopefully, to stratify patients enrolled in second-line treatment future trials. The findings made in the present trial may thus represent a new benchmark of nitrosourea activity in a homogeneously pre-treated population that failed to respond to the new standard treatment.

\section{References}

1. Avril MF, Aamdal S, Grob JJ, Hauschild A, Mohr P, Bonerandi JJ, Weichenthal M, Neuber K, Bieber T, Gilde K, Guillem Porta V, Fra J, Bonneterre J, Saiag P, Kamanabrou D, Pehamberger H, Sufliarsky J, Gonzalez Larriba JL, Scherrer A, Menu Y (2004) Fotemustine compared with dacarbazine in patients with disseminated malignant melanoma: a phase III study. J Clin Oncol 22:1118-1125

2. Brandes AA, Basso U, Pasetto LM, Ermani M (2001) New strategy developments in brain tumor therapy. Curr Pharm Des 7:1553-1580

3. Brandes AA, Tosoni A, Amista P, Nicolardi L, Grosso D, Berti F, Ermani M (2004) How effective is BCNU in recurrent glioblastoma in the modern era? A phase II trial. Neurology 63:1281-1284

4. Brandes AA, Tosoni A, Cavallo G, Bertorelle R, Gioia V, Franceschi E, Biscuola M, Blatt V, Crino L, Ermani M (2006) Temozolomide 3 weeks on and 1 week off as first-line therapy for recurrent glioblastoma: phase II study from gruppo italiano cooperativo di neuro-oncologia (GICNO). Br J Cancer 95:1155-1160

5. Brandes AA, Tosoni A, Vastola F, Pasetto LM, Coria B, Danieli D, Iuzzolino P, Gardiman M, Talacchi A, Ermani M (2004) Efficacy and feasibility of standard procarbazine, lomustine, and vincristine chemotherapy in anaplastic oligodendroglioma and oligoastrocytoma recurrent after radiotherapy. A Phase II study. Cancer 101:2079-2085

6. CBTRUS CBtrotUS (2004) Primary brain tumors in the United States. Statistical report 1997-2001

7. Esteller M, Garcia-Foncillas J, Andion E, Goodman SN, Hidalgo OF, Vanaclocha V, Baylin SB, Herman JG (2000) Inactivation of the DNA-repair gene MGMT and the clinical response of gliomas to alkylating agents. N Engl J Med 343:1350-1354

8. Fabrini MG, Silvano G, Lolli I, Perrone F, Marsella A, Scotti V, Cionini L (2008) A multi-institutional phase II study on second-line 
Fotemustine chemotherapy in recurrent glioblastoma. J Neurooncol. doi:10.1007/s11060-008-9739-6

9. Filippeschi S, Colombo T, Bassani D, De Francesco L, Arioli P, D'Incalci M, Bartosek I, Guaitani A (1988) Antitumor activity of the novel nitrosourea S10036 in rodent tumors. Anticancer Res 8:1351-1354

10. Fischel JL, Formento P, Etienne MC, Gioanni J, Frenay M, Deloffre P, Bizzari JP, Milano G (1990) In vitro chemosensitivity testing of Fotemustine (S 10036), a new antitumor nitrosourea. Cancer Chemother Pharmacol 25:337-341

11. Hegi ME, Diserens AC, Godard S, Dietrich PY, Regli L, Ostermann S, Otten P, Van Melle G, de Tribolet N, Stupp R (2004) Clinical trial substantiates the predictive value of $O$-6-methylguanine-DNA methyltransferase promoter methylation in glioblastoma patients treated with temozolomide. Clin Cancer Res 10:1871-1874

12. Hegi ME, Diserens AC, Gorlia T, Hamou MF, de Tribolet N, Weller M, Kros JM, Hainfellner JA, Mason W, Mariani L, Bromberg JE, Hau P, Mirimanoff RO, Cairncross JG, Janzer RC, Stupp R (2005) MGMT gene silencing and benefit from temozolomide in glioblastoma. N Engl J Med 352:997-1003

13. Herman JG, Graff JR, Myohanen S, Nelkin BD, Baylin SB (1996) Methylation-specific PCR: a novel PCR assay for methylation status of CpG islands. Proc Natl Acad Sci USA 93:9821-9826

14. Khayat D, Lokiec F, Bizzari JP, Weil M, Meeus L, Sellami M, Rouesse J, Banzet P, Jacquillat C (1987) Phase I clinical study of the new amino acid-linked nitrosourea, S 10036, administered on a weekly schedule. Cancer Res 47:6782-6785

15. Kleeberg UR, Engel E, Israels P, Brocker EB, Tilgen W, Kennes C, Gerard B, Lejeune F, Glabbeke MV, Lentz MA (1995) Palliative therapy of melanoma patients with fotemustine. Inverse relationship between tumour load and treatment effectiveness. A multicentre phase II trial of the EORTC-melanoma cooperative group (MCG). Melanoma Res 5:195-200

16. Levin VA (1980) Relationship of octanol/water partition coefficient and molecular weight to rat brain capillary permeability. J Med Chem 23:682-684

17. Macdonald DR, Cascino TL, Schold SC Jr, Cairncross JG (1990) Response criteria for phase II studies of supratentorial malignant glioma. J Clin Oncol 8:1277-1280

18. Meulemans A, Giroux B, Hannoun P, Robine D, Henzel D (1991) Comparative diffusion study of two nitrosoureas: carmustine and fotemustine in normal rat brain, human, and rat brain biopsies. Chemotherapy 37:86-92

19. Mornex F, Thomas L, Mohr P, Hauschild A, Delaunay MM, Lesimple T, Tilgen W, Bui BN, Guillot B, Ulrich J, Bourdin S, Mousseau M, Cupissol D, Bonneterre ME, De Gislain C, Bensadoun RJ, Clavel M (2003) A prospective randomized multicentre phase III trial of fotemustine plus whole brain irradiation versus fotemustine alone in cerebral metastases of malignant melanoma. Melanoma Res 13:97-103
20. Ozkan M, Altinbas M, Er O, Kaplan B, Coskun HS, Karahacioglu E, Menku A, Cihan Y, Kontas O, Akdemir H (2004) Post-operative sequential chemo-radiotherapy in high-grade cerebral gliomas with fotemustine. J Chemother 16:298-302

21. Palmisano WA, Divine KK, Saccomanno G, Gilliland FD, Baylin SB, Herman JG, Belinsky SA (2000) Predicting lung cancer by detecting aberrant promoter methylation in sputum. Cancer Res 60:5954-5958

22. Perry J, Mason W, Belanger K, Kavan P, Fulton D, Easaw J, Kirby S, Macdonald D, Shields C, JFP (2008) The Temozolomide RESCUE study: a Phase II trial of continuous (28/28) dose-intense temozolomide (TMZ) after progression on conventional 5/28 day TMZ in patients with recurrent malignant glioma. In: 8th Congress of European Association of Neurooncology

23. Perry JR, Rizek P, Cashman R, Morrison M, Morrison T (2008) Temozolomide rechallenge in recurrent malignant glioma by using a continuous temozolomide schedule: the "rescue" approach. Cancer 113:2152-2157

24. Raymond E, Haon C, Boaziz C, Coste M (1996) Logistic regression model of fotemustine toxicity combining independent phase II studies. Cancer 78:1980-1987

25. Schallreuter KU, Wenzel E, Brassow FW, Berger J, Breitbart EW, Teichmann W (1991) Positive phase II study in the treatment of advanced malignant melanoma with fotemustine. Cancer Chemother Pharmacol 29:85-87

26. Scoccianti S, Detti B, Sardaro A, Iannalfi A, Meattini I, Leonulli BG, Borghesi S, Martinelli F, Bordi L, Ammannati F, Biti G (2008) Second-line chemotherapy with fotemustine in temozolomide-pretreated patients with relapsing glioblastoma: a single institution experience. Anticancer Drugs 19:613-620

27. Stupp R, Mason WP, van den Bent MJ, Weller M, Fisher B, Taphoorn MJ, Belanger K, Brandes AA, Marosi C, Bogdahn U, Curschmann J, Janzer RC, Ludwin SK, Gorlia T, Allgeier A, Lacombe D, Cairncross JG, Eisenhauer E, Mirimanoff RO (2005) Radiotherapy plus concomitant and adjuvant temozolomide for glioblastoma. N Engl J Med 352:987-996

28. van Engeland M, Weijenberg MP, Roemen GM, Brink M, de Bruine AP, Goldbohm RA, van den Brandt PA, Baylin SB, de Goeij AF, Herman JG (2003) Effects of dietary folate and alcohol intake on promoter methylation in sporadic colorectal cancer: the Netherlands cohort study on diet and cancer. Cancer Res 63:3133-3137

29. Vredenburgh JJ, Desjardins A, Herndon JE 2nd, Marcello J, Reardon DA, Quinn JA, Rich JN, Sathornsumetee S, Gururangan S, Sampson J, Wagner M, Bailey L, Bigner DD, Friedman AH, Friedman HS (2007) Bevacizumab plus irinotecan in recurrent glioblastoma multiforme. J Clin Oncol 25:4722-4729

30. Wick A, Felsberg J, Steinbach JP, Herrlinger U, Platten M, Blaschke B, Meyermann R, Reifenberger G, Weller M, Wick W (2007) Efficacy and tolerability of temozolomide in an alternating weekly regimen in patients with recurrent glioma. J Clin Oncol 25:3357-3361 